\title{
Accidentes de tránsito y el consumo de alcohol en una unidad de urgencia de La Paz, Bolivia
}

\author{
Inês Magali Pelaez Mariscal ${ }^{1}$ \\ Edilaine Cristina da Silva²
}

\begin{abstract}
Estudio de tipo cuantitativo, descriptivo, observacional y transversal. El objetivo del estudio fue conocer la distribución y características de los accidentes de tránsito relacionados con el consumo de alcohol en una unidad de urgencias de un hospital de clínicas de La Paz, en Bolivia. La muestra estuvo conformada por 62 personas, choferes y peatones, mayores de 15 años, que estuvieron involucrados en accidentes de tránsito por estado de ebriedad, los cuales acudieron a la Unidad de Urgencias. Fue aplicado una encuesta elaborado por los investigadores para recolectar datos sociodemográficos y de morbilidad de las víctimas y datos que evalúan el consumo de alcohol del conductor responsable del accidente. Los resultados evidenciaron que $71 \%$ de los accidentados eran hombres; $32,3 \%$ presentaban edades entre 21 y 30 años; $29 \%$ eran estudiantes y $25.8 \%$ eran choferes. El estudio permitió la caracterización de las personas accidentadas, del consumo de alcohol e del accidente.
\end{abstract}

Descriptores: Alcoholismo; Accidentes de Tránsito; Prevención de Accidentes.

\footnotetext{
${ }^{1}$ Maestría en Educación Superior, Profesor de la Carrera de Enfermería, Universidad Mayor de San Andrés, Bolivia. E-mail: inespelaezm@yahoo.es.

${ }^{2}$ Enfermera, Doctor en Enfermería, Profesor Doctor, Escola de Enfermagem de Ribeirão Preto, Universidade de São Paulo, Centro Colaborador de la OMS para el Desarrollo de la Investigación en Enfermería, SP, Brasil. E-mail: nane@eerp.usp.br.
}

Correspondencia:

Edilaine Cristina da Silva

Universidade de São Paulo. Escola de Enfermagem de Ribeirão Preto

Av. Bandeirantes, 3900

Bairro Monte Alegre

CEP: 14040-902 Ribeirão Preto, SP, Brasil

E-mail: nane@eerp.usp.br 


\section{Acidentes de trânsito e o consumo de álcool em unidade de urgência de La Paz, Bolívia}

Este é um estudo tipo quantitativo, descritivo, observacional, transversal. O objetivo foi conhecer a distribuição e as características dos acidentes de trânsito relacionados ao consumo de álcool em unidade de urgência de um hospital de clínicas de La Paz, Bolívia. A amostra foi constituída por 62 pessoas, motoristas e pedestres, maiores de 15 anos, envolvidos em acidentes de trânsito por estado de embriaguez, atendidos na unidade de urgência. Foi aplicado um questionário, elaborado pelos pesquisadores, para coletar dados sociodemográficos e de morbidade da vítima, e dados que avaliam o consumo de álcool do condutor responsável pelo acidente. Os resultados evidenciaram que $71 \%$ dos acidentados eram homens, 32,3\% apresentavam idade entre 21 e 30 anos, 29\% eram estudantes e $25,8 \%$ eram motoristas. O estudo permitiu a caracterização das pessoas acidentadas, do consumo de álcool e do acidente.

Descritores: Alcoolismo; Acidentes de Trânsito; Prevenção de Acidentes.

\section{Traffic accidents and alcohol consumption in an emergency unit of La Paz, Bolivia}

The aim of this quantitative, descriptive, observational, cross-sectional study was to characterize the distribution and characteristics of motor vehicle accidents related to alcohol consumption. The sample was composed of 62 drivers and pedestrians, over 15 years old, who were involved in motor vehicle accidents involving alcohol, and were attended at the Emergency Unit of the Clinical Hospital of La Paz - Bolivia. A survey designed by the researchers to collect partner-demographic data and morbidity of the victim was applied and data that evaluated the alcohol consumption of the driver responsible for the accident was collected. The results demonstrated that $71 \%$ of the victims were men; $32.3 \%$ were between 21 and 30 years old; $29 \%$ were students and $25.8 \%$ were the drivers. This study shows the characterization of victims, alcohol consumption and accidents.

Descriptors: Alcoholism; Accidents, Traffic; Accident Prevention.

\section{Introducción}

La Organización Mundial de la Salud (OMS) estima que los accidentes de tránsito son responsables por un 1,2 millones de muertes y 50 millones de personas sufren serias lesiones a cada año, en el mundo, siendo la novena causa de mortalidad, correspondiendo al 2,2\% de muertes en el ámbito mundial. Las proyecciones para el año 2.020 apuntan que estos accidentes ocuparan el tercer lugar entre las causas de mortalidad. Cada 30 segundos muere una persona por esta causa y por cada muerte hay 34 heridos, esto seguirá en aumento si no se toman medidas de acción por lo que es necesario prevenirlos y actuar prontamente(1).

En los países de América Latina y el Caribe mueren cada año más de 130.000 personas, más de
1.200.000 sufren heridas y cientos de miles quedan discapacitados a consecuencia de las colisiones $y$ atropellamientos en la vía pública por el consumo de alcohol, aunque es difícil establecer comparaciones entre los diversos estudios realizados, debido a las grandes variaciones que existen, de un país para otro, en los límites legales de alcoholemia impuestos por la legislación local(2).

Bolivia tiene aproximadamente 9.024,922 habitantes, la prevalecía del consumo de alcohol en personas mayores de 12 años de edad es de 42,5\% lo que equivale a 1.390 .172 personas. Esto implica que uno de cada dos bolivianos consume habitualmente una bebida alcohólica ${ }^{(3-4)}$. 
Según estimativas realizadas por el Ministerio de Salud y Deportes en Bolivia, 55 accidentes se producen por día y 2,5 por hora, con las complicaciones de gastos, muertes, heridos y discapacitados. Por lo tanto representa una cuestión social no solo por las pérdidas de vida, principalmente entre los jóvenes, sino también por los costos directos e indirectos, demostrando, de esa forma un significativo peso económico, social y de sufrimiento de las familias ${ }^{(5-6)}$.

El Departamento de La Paz cuenta con 2.544,864 habitantes $/ \mathrm{Km}^{2}$ y el Municipio de La Paz con 793.293 habitantes $/ \mathrm{Km}^{2}$, tiene una tasa de mortalidad por accidente de tránsito muy por encima de la Nacional, esa tasa alcanza 12 por cada 100.000 habitantes, en La Paz; número de muertes y heridos que sobrepasa a otros departamentos; las personas heridas en accidentes de tránsito ocurridos en La Paz, Santa Cruz y Cochabamba representan más del $70 \%$ de todos los heridos registrados en los últimos cinco años en Bolivia, la mayoría corresponde al sexo masculino, en edad económicamente productiva( ${ }^{(3,7)}$.

Según los datos de la Policía del Organismo Operativo de Tránsito en la ciudad de La Paz, en 2.003 fue el estado de embriaguez la tercera causa de accidentes de tránsito con un $16.34 \%$ del total de casos registrados por distintas causas. En la actualidad se ubica como la segunda causa con el $22.9 \%$, aspecto que demuestra un incremento considerable en relación a los años anteriores ${ }^{(8-9)}$.

Estos datos además de expresar la situación de los accidentes de tránsito en general y los relacionados con el consumo de alcohol en Bolivia y específicamente en la ciudad de La Paz, crea la necesidad de abordar, específicamente, los accidentes de tránsito, el consumo de bebidas alcohólicas y el efecto o trascendencia sobre la vida y salud de los afectados.

El Ministerio de la Salud en Bolivia recomienda investigar la temática y considerar temas como: costo en dinero que afecta a las víctimas, la discapacidad por accidentes de tránsito, la identificación de segmentos poblacionesdevíctimas, intervalosdeedad, características de los conductores, entre otros. Estos aportes ayudarían a fortalecer las estrategias para planificar e implementar la seguridad en las vías de tránsito de Bolivia(7) Como consecuencia del impacto de esta problemática se va incrementando las investigaciones direccionadas a la temática ${ }^{(10-11)}$.

La no existencia de un estudio en Bolivia sobre los accidentes de tránsito relacionados al consumo de alcohol y su efecto en las lesiones físicas, lleva a considerar la importancia de estudios que incrementen informaciones sobre esta temática en la ciudad de La Paz, en Bolivia.

\section{Objetivos}

Conocer la distribución y características del aparecimiento de accidentes de tránsito relacionados con el consumo de alcohol en la Unidad de Urgencias del Hospital de Clínicas La Paz - Bolivia, en el periodo de febrero a abril 2007.

\section{Metodología}

\section{Tipo de Estudio y variables}

El presente trabajo de investigación es de tipo cuantitativo, descriptivo, observacional y transversal en el tiempo, comprendido entre los meses de febrero a abril de 2007. Se buscó destacar los intervalos de edad afectados, género, tipo de accidente, horario, tipo de consumo de bebida, frecuencia de ocurrencia, magnitud de morbilidad en cuanto a lesiones físicas y su gravedad.

\section{Local y Muestra}

El estudio fue realizado en una unidad de urgencias de un hospital de clínicas en La Paz, Bolivia. La muestra está conformada por 62 personas, todos los choferes y peatones mayores de 15 años involucrados en accidentes de tránsito en estado de ebriedad, que acudieron a la unidad para recibir asistencia inmediata. Se excluyó a todos los demás casos atendidos en la unidad de urgencias por diferentes causas.

\section{Instrumentos}

Entrevistadores entrenados y orientados aplicaron una encuesta elaborada por los investigadores para recolectar los datos, cuyo tiempo de aplicación era de 10 minutos.

El instrumento aplicado constaba de 3 partes: datos sociodemográficos de la víctima, datos de ocurrencia de morbilidad y mortalidad y datos del conductor responsable del accidente. Fue elaborado buscándose lograr el objetivo del estudio, siendo considerado válido por investigadores en la temática. Hay que aclarar que el instrumento debería ser corto y de fácil aplicación debido a la característica dinámica del servicio de urgencias.

Los entrevistadores recibieron orientaciones generales sobre como portarse en las encuestas, sobre el objetivo de cada una de las cuestiones del instrumento y sobre el llenado del instrumento. 


\section{Consideraciones Éticas}

El proyecto fue presentado a la Universidad Mayor de San Andrés, Carrera de Enfermería y a la Unidad de Emergencias del Hospital de Clínicas y se obtuvo la autorización del Jefe de la Unidad de Emergencias y se mantuvo coordinación con la Jefa de Enfermeras de la unidad para aplicar el instrumento previo consentimiento informado de los pacientes.

\section{Análisis de los datos}

El análisis de los resultados se efectuó con el paquete estadístico SPSS 10.0. Se procedió al estudio descriptivo observando la distribución de los datos respecto a su naturaleza.

\section{Resultados}

Los individuos accidentados eran en su mayoría hombres (71\%) con edad de 21 a 30 años (27,4\%). Entre las mujeres, el intervalo de edad que sufrió más incidentes fue el que estaba 16 y 20 años (12,9\%). Del total, 43,5\% de los accidentados eran solteros, siendo que entre los hombres había más casados y entre las mujeres más solteras, $30,6 \%$ y $16,1 \%$, respectivamente (Tabla 1).

Tabla 1 - Descripción de las variables sociodemográficas de las víctimas de accidentes de tránsito según el género, La Paz, Bolivia, 2007

\begin{tabular}{|c|c|c|c|c|c|c|}
\hline \multirow{2}{*}{$\begin{array}{l}\text { Variables } \\
\qquad(n=62)\end{array}$} & \multicolumn{2}{|c|}{ Hombres } & \multicolumn{2}{|c|}{ Mujeres } & \multicolumn{2}{|c|}{$\begin{array}{c}\text { Frecuencia } \\
\text { Total }\end{array}$} \\
\hline & $\mathbf{N}$ & $\%$ & $\mathbf{N}$ & $\%$ & $\mathbf{N}$ & $\%$ \\
\hline \multicolumn{7}{|l|}{ Edad } \\
\hline 16 a 20 & 1 & 1,6 & 8 & 12,9 & 9 & 14,5 \\
\hline 21 a 30 & 17 & 27,4 & 3 & 4,8 & 20 & 32,3 \\
\hline 31 a 40 & 5 & 8,1 & 3 & 4,8 & 8 & 12,9 \\
\hline 41 a 50 & 9 & 14,5 & 2 & 3,2 & 11 & 17,7 \\
\hline$>50$ & 12 & 19,4 & 2 & 3,2 & 14 & 22,6 \\
\hline \multicolumn{7}{|l|}{ Estado Civil } \\
\hline Soltero & 17 & 27,4 & 10 & 16,1 & 27 & 43,5 \\
\hline Casado & 19 & 30,6 & 4 & 6,4 & 23 & 37,1 \\
\hline Divorciado & 1 & 1,6 & 1 & 1,6 & 2 & 3,2 \\
\hline Viudo & 1 & 1,6 & 1 & 1,6 & 2 & 3,2 \\
\hline Concubino & 6 & 9,7 & 2 & 3,2 & 8 & 13,0 \\
\hline \multicolumn{7}{|l|}{ Actividad Profesional } \\
\hline Estudiante & 8 & 12,9 & 10 & 16,1 & 18 & 29.0 \\
\hline Empleado público & 9 & 14,5 & 2 & 3,2 & 11 & 17.7 \\
\hline Comerciante & 5 & 8,1 & 2 & 3,2 & 7 & 11.3 \\
\hline Chofer & 16 & 25,8 & 0 & 0,0 & 16 & 25.8 \\
\hline Sin ocupación & 6 & 9,7 & 4 & 6,4 & 10 & 16.2 \\
\hline Total & 44 & 71,0 & 18 & 29,0 & 62 & 100,0 \\
\hline
\end{tabular}

Los datos de actividad profesional indicaron que entre las víctimas de accidentes el mayor porcentaje era de estudiantes (29\%) seguido de los choferes (25,8\%). La totalidad de los choferes era de hombres; en cuanto a las mujeres, fueron las estudiantes las más afectadas $(16,1 \%)$. Los solteros, en ambos géneros, fueron los más afectados con el 29\% (Tabla 1 ).

Los atropellamientos fueron el tipo de accidente predominante con el 53,2\%, seguido de las colisiones o choques con el 38,7\% (Tabla 2).

Los casos atendidos en el servicio de urgencias fueron accidentes procedentes de la zona urbana: avenidas $(45,2 \%)$ y calles $(32,3 \%)$ (Tabla 2$)$.

Tabla 2 - Distribución de las víctimas por las variables del accidente y del accidentado de tránsito, La Paz, Bolivia, 2007

\begin{tabular}{|c|c|c|}
\hline Variable $(n=62)$ & Frecuencia Total & $\%$ \\
\hline \multicolumn{3}{|l|}{ Tipo de Accidente } \\
\hline Atropello (A) & 33 & 53,2 \\
\hline Colisiones (C) & 24 & 38,7 \\
\hline Arrollamiento (AR) & 3 & 4,8 \\
\hline Vuelco (V) & 2 & 3,2 \\
\hline \multicolumn{3}{|l|}{ Sitio del Accidente } \\
\hline Avenida & 28 & 45,2 \\
\hline Calle & 20 & 32,3 \\
\hline Carretera & 6 & 9,7 \\
\hline Ciudad Del Alto & 8 & 12,8 \\
\hline \multicolumn{3}{|l|}{ Gravedad del Accidentado } \\
\hline Leve (dado de alta) & 34 & 54,8 \\
\hline Moderado (internado) & 12 & 19,4 \\
\hline Grave (cirugía) & 16 & 25,8 \\
\hline \multicolumn{3}{|c|}{ Diagnóstico Médico del Accidentado } \\
\hline Policontusión & 37 & 59,7 \\
\hline Fractura & 18 & 29,0 \\
\hline Trauma Cráneo-Encefálico & 7 & 11,3 \\
\hline \multicolumn{3}{|l|}{ Responsable del Accidente } \\
\hline Peatón & 24 & 38,7 \\
\hline Chofer & 38 & 61,2 \\
\hline \multicolumn{3}{|l|}{ Día del Accidente } \\
\hline Lunes & 4 & 6,4 \\
\hline Martes & 12 & 19,3 \\
\hline Miércoles & 2 & 3,2 \\
\hline Jueves & 3 & 4,8 \\
\hline Viernes & 11 & 17,7 \\
\hline Sábado & 16 & 25,8 \\
\hline Domingo & 14 & 22,6 \\
\hline \multicolumn{3}{|l|}{ Horario del Accidente } \\
\hline Mañana & 5 & 8,1 \\
\hline Tarde & 20 & 32,3 \\
\hline Noche & 26 & 41,9 \\
\hline Madruga & 11 & 17,7 \\
\hline \multicolumn{3}{|l|}{ Condición del Conductor } \\
\hline Con Consumo & 38 & 61,3 \\
\hline Sin Consumo & 24 & 38,7 \\
\hline
\end{tabular}


El $54,8 \%$ de los casos atendidos en el servicio de urgencias por accidentes de tránsito de conductores en estado de ebriedad fueron de condición leve según el diagnostico médico policontusiones $(59,7 \%)$, los cuales estuvieron en observación y dados de alta en las primeras 24 horas. Sin embargo los fracturados correspondieron al $29 \%$ y los de condición grave al $25,8 \%$, quienes tuvieron que ser ingresados en el servicio de la especialidad para intervención quirúrgica, siendo mayor el daño físico para estas personas.

Los responsables del accidente que se encontraban en estado de ebriedad con mayor frecuencia eran los choferes $(61,2 \%)$, en comparación de los peatones ebrios con el 38,7\% (Tabla 2)

Existieron, en los tres meses de estudio, considerablemente más accidentes con personas en estado de ebriedad, los fines de semana; en orden de frecuencia el día sábado, domingo y viernes $(25,8 ; 22,6$; $17.7 \%)$. Llama la atención la frecuencia encontrada el día martes (19,3\%).

El horario de mayor ocurrencia encontrada fue la noche $(41,9 \%)$, seguido en importancia el correspondiente a la tarde $(32,3 \%)$ y finalmente el de la madrugada $(17,7 \%)$.

Respecto a los conductores en estado de ebriedad, se pudo observar que el conductor masculino tenía la mayor frecuencia de estar implicado en un accidente de tránsito relacionado al consumo de alcohol y se observó nuevamente que son los hombres comprendidos entre las edades de 21 y 30 años los más implicados (60,5\%). De estos el 63,1\% refirieron que sufrieron el accidente por la primera, el encuestado demostró sentir dudas e incomodidad.

A respecto del tipo de bebida alcohólica consumida, el $36,8 \%$ tenía preferencia por la cerveza y un número similar $(36,8 \%)$ combinaban todo tipo de bebidas (cerveza, singani, ron e inclusive alcohol puro). El 68,4\% consumieron más de una botella. En general la mayoría de las personas consumió cantidades y concentraciones altas de alcohol en sus diversos tipos.

El $47,4 \%$ de los conductores eran propietarios y el $15,8 \%$ asalariado, de los cuales el $63,2 \%$ contaba con seguro obligatorio para accidentes de tránsito y un $18,4 \%$ no tenía seguro. No contestaron el restante de los conductores $18,4 \%$.

\section{Discusión}

Según los resultados de esta investigación, los conductores en estado de ebriedad implicados en accidentes de tránsito eran todos del género masculino, pero además este grupo de personas tenía la características de ser jóvenes en edad productiva, comprendidos entre 21 y 30 años, choferes, consumidores de bebidas alcohólicas, particularmente los fines de semana, por la noche, donde combinan diversos tipos de bebidas e inclusive refirieron hacer uso de alcohol puro; como segunda opción eligen de preferencia la cerveza, en cantidades mayores a una botella $(63,4 \%$ del total de conductores). Esto indica que las dosis en estas personas excedieron el límite permitido por la Policía de Tránsito en Bolivia y también la dosis límite permitida por la OMS de $0,57 \mathrm{~g}$ de alcohol por litro de sangre, aunque la OMS complementa que es difícil establecer comparaciones entre los límites legales de alcoholemia y su aplicación existentes por los diversos países de Latinoamérica(12).

Se pudo observar en el servicio de Urgencias que todos los casos atendidos relacionados al consumo de alcohol de accidentes de tránsito que envolvieron personas en estado de ebriedad son la segunda causa de ingreso, haciendo el 19,9\% del total, un promedio de 4 a 5 casos atendidos después de las intoxicaciones alcohólicas superando las estimativas de la OMS, que ubica en el tercer lugar a los accidentes de tránsito como causa de enfermedad o lesión. A esto se agrega el factor de consumo de alcohol por parte de los peatones, puesto que según investigaciones realizadas, indican que uno de cada dos bolivianos consume alcohol, lo cual aumenta el riesgo(1,4).

Por otra parte llama la atención la participación de mujeres jóvenes menores de 19 años (12,9\%), en calidad de estudiantes (16.1\%), como víctimas en estado de ebriedad, aspecto que difiere de otro estudio efectuado, el que afirma que la mayoría de hombres y mujeres en esa edad, particularmente de 25 a 30 años, moderan su consumo, mientras que los alcohólicos aumentan sus dificultades sufriendo los primeros problemas importantes con el alcohol entre los 24 y 45 años $^{(13)}$. En ese sentido, para este importante intervalo de edad, se tiene que trabajar en forma preventiva, en tareas conjuntas e interdisciplinarias, para conocer primeramente los factores de consumo y actuar prontamente sobre los mismos, puesto que son individuos en edades de reproducción y de productividad laboral e intelectual que deben ser protegidos.

De acuerdo a los registros del Organismo Operativo de Tránsito en cooperación con el Ministerio de Salud de Bolivia, en 2004 las colisiones son la primera causa de accidentes (50,35\%), seguida por colisiones con objeto fijo o autos parados $(23,18 \%)$, y la tercera causa 
corresponde a los atropellamientos $(20,33 \%)^{(4)}$. Esta distribución es diferente a lo encontrado en la unidad de urgencias en la que el tipo de accidente más frecuente fue el de atropellos $(53,2 \%)$, seguido por las colisiones $(38,7 \%)$. Probablemente esto tenga relación con el consumo de alcohol por parte de los peatones 38,7\% y el hecho del elevado consumo de alcohol hecho por los bolivianos $^{(4)}$, sin embargo estas víctimas son atendidas en los diferentes servicios de salud y sometidas a procedimientos invasores complejos en la búsqueda de sobrevivencia(14).

La frecuencia encontrada de personas accidentadas en estado de ebriedad el día martes se destaca de los otros días de la semana; la misma tiene relación con el feriado de carnavales en el mes de febrero y particularmente este día se lleva adelante por costumbre y tradición la Challa* de los bienes inmuebles de los propietarios, con alcohol y consumo del mismo.

\section{Consideraciones Finales}

El análisis de los accidentes de tránsito, con personas en estado de ebriedad en Bolivia y en los servicios de urgencia, se encuentra limitado por la imposibilidad de identificar los accidentes que se definen como tales. Esto se debe, principalmente a la ausencia de registro de la alcoholemia o de prueba efectiva del consumo en los servicios de salud, en general por falta de recursos materiales y personal capacitado para este procedimiento, resultando en que un porcentaje de los implicados no son detectados.

En realidad, los accidentes relacionados al consumo de alcohol, identificado en el servicio de urgencias del hospital de clínicas, no representan la totalidad de dichos casos, pero constituyen una referencia importante para poder generar hipótesis, comparar y conocer más a fondo los aspectos estudiados. Sin embargo, a pesar de las limitaciones, se obtuvieron datos que son relevantes en función del objetivo perseguido. Los resultados permitieron conocer el perfil y algunas de las características de las personas, el consumo y el accidente; datos que serán útiles e importantes para que la enfermería pueda desarrollar programas educativos en esa temática, además de inducir y generar conductas preventivas continuas para lo cual es necesario aunar esfuerzos conjuntos interdisciplinarios e intersectoriales para realizar un abordaje integral e holístico de los casos, con la finalidad de prevenir accidentes y lesiones por estado de ebriedad en el tránsito de La Paz, Bolivia.

\section{Agradecimientos}

Agradecemos a la Comisión Interamericana para el Control del Abuso de Drogas/CICAD de la Secretaria de Seguridad Multidimensional/SSM de la Organización de los Estados Americanos/OEA, la Secretaria Nacional de Políticas sobre Drogas/SENAD do Gabinete de Seguridad Institucional/Brasil, la Escuela de Enfermería de Ribeirao Preto de la Universidad de Sao Paulo y Centro Colaborador de la Organización Mundial de la Salud para el Desarrollo de la Investigación en Enfermería, la población representada en los estudios de investigación, bien como a las autoridades de las universidades representadas por los participantes del Programa En-Line de Especialización en Investigación sobre el Fenómeno de las Drogas PREINVEST, periodos 2005, 2006, 2007 y 2008.

\section{Referencias}

1. Organización Mundial de la Salud. Informe mundial sobre prevención de los traumatismos causados por el transito. Ginebra: Organización Mundial de la Salud; 2004.

2. World Health Organization. Facts about injuries. WHO; 2002. [acceso en: noviembro 2006]. Disponible en: http://www.who. en/violencia_injury-prevencion/indez.html.

3. Instituto Nacional de Estadística. Informe Censo Poblacional. Bolivia: INE; 2003.

4. Centro Latinoamericano de Investigación Científica (CELINBolivia). La prevalencía del consumo de alcohol. Bolívia: CELINBolivia; 2005.
5. Ministerio de Salud y Deportes (MSD), Organización Panamericana de la Salud/ Organización Mundial de la Salud, Sociedad Médica de Salud Pública de Cochabamba. Memorias del taller de prevención de accidentes de transito, del 26 y 27 de Febrero de 2001. Cochabamba: OPS/OMS; 2001.

6. Organización Panamericana de la Salud/Organización Mundial de la Salud (BO). La seguridad vial no es accidental: taller para el día mundial de la salud 2004. La Paz; 2004.

7. Ministerio de Salud y Deportes (MSD), Policía Nacional, Organización Panamericana de la Salud/ Organización Mundial de la Salud. Accidentes de Transito en Bolivia situación en el último quinquenio. La Paz (BO): Ministério de Salud y Deportes; 2004.

\footnotetext{
* Challa: tradición andina, en Bolivia el martes de carnaval la población hace ofrenda a la Pachamama - diosa de la Madre Tierra - con el derrame de alcohol, vino u otra bebida alcohólica, dulces, frutas, mezcla, serpentina, flores a sus propiedades, casas, bienes inmuebles y todo bien, para que tenga prosperidad y en agradecimiento a la Pachamama, en ese acto la gente consume bebidas alcohólicas ese día y todos los demás de carnaval.
} 
8. División Operativa de Transito Central (DOTC). Situación de los accidentes de transito últimos 5 años Bolivia1999/2003. Bolivia: División Operativa de Transito Central; 2004.

9. División Operativa de Transito Departamental (DOTD). Causas frecuentes de hechos de transito 2000/2006. La Paz, División Operativa de Transito Departamental; 2007.

10. Oliveira MR, Luis MAV. Factores de riesgo para el consumo de alcohol en escolares de 10 a 18 años, de establecimientos educativos fiscales en la ciudad de La Paz - Bolivia (2003 2004). Rev. Latino-Am. Enfermagem. October 2005; 13 (no. spe):880-7.
11. Salcedo LJA, Carvalho AMP. Maltrato infantil por agresores bajo efecto del alcohol. Rev. Latino-Am. Enfermagem. October 2005; 13(no.spe):827-35.

12. Sharmam $\mathrm{CH}$. OPS. El problema del alcohol. Perspec de Salud OPAS 2005; 10(1) Disponible en: URL: http://www.paho. org/Spanish/DD/PIN/Numero21_articulo04.htm

13. Fuchs CS, Stampfer MJ, Colditz GA, Giovannucci EL, Manson JE, Kawachi I, et al. Alcohol consumption and mortality among women. N Engl J Méd. 1995; 332:1245-50.

14. Malvestio MAA, Sousa RMC. Análise do valor predeterminante dos procedimentos da fase pré-hospitalar na sobrevivência das vítimas de trauma. Rev. Latino-Am. Enfermagem, $2008 ; 16(3): 432-8$. 